\title{
TO EVALUATE THE SENSITIVITY AND SPECIFICITY OF CSF-ADA AS A DIAGNOSTIC TOOL IN TUBERCULOSIS MENINGITIS
}

\author{
Manish Kumar Singh¹, K. Chandran², Shrawan Kumar³, Pranjal Pankaj ${ }^{4}$, Ishan Parasher ${ }^{5}$
}

\section{HOW TO CITE THIS ARTICLE:}

Manish Kumar Singh, K. Chandran, Shrawan Kumar, Pranjal Pankaj, Ishan Parasher. "To evaluate the sensitivity and specificity of CSF-ADA as a diagnostic tool in tuberculosis meningitis". Journal of Evolution of Medical and Dental Sciences 2013; Vol2, Issue 32, August 12; Page: 5966-5970.

\begin{abstract}
INTRODUCTION: Tuberculosis remains one of the world's deadliest diseases \& tubercular meningitis is one of the most deadly complications due to missed diagnosis and delayed treatment result in significant morbidity and mortality. The signs and symptoms, results of routine analysis of CSF and radiographic findings for patients with CNS tuberculosis are often inadequate in making a definitive diagnosis. AIMS AND OBJECTIVES: 1. To study the levels of Adenosine deaminase in cases of meningitis, 2. To evaluate the sensitivity and specificity of CSF-ADA as a diagnostic test in tuberculosis meningitis. MATERIAL AND METHODS: In the cross sectional study, Adenosine deaminase activity (ADA) was studied in cerebrospinal fluid of 60 cases of tuberculous meningitis, 10 cases of pyogenic meningitis. 10 cases of aseptic meningitis and15 control. RESULTS: The mean CSF-ADA activity was 14.1 \pm 1.96 (tuberculous meningitis); $4.92 \pm 1.27$ (pyogenic meningitis); $3.66 \pm 1.03$ (aseptic meningitis) and 1.69 $\pm 0.44 \mathrm{U} / \mathrm{l}$ (control) respectively. The sensitivity and specificity of this test for diagnosis of tuberculous meningitis was $100 \%$ and $97.44 \%$ respectively with ADA value of more than $10 \mathrm{U} / \mathrm{L}$. CONCLUSION: The adenosine deaminase activity in tuberculous meningitis cases was significantly higher. So it can be of great value in the early and more definitive diagnosis of tuberculous meningitis, to help early diagnosis and treatment to prevent from deadly complications of tubercular meningitis.
\end{abstract}

KEY WORDS: Tuberculous meningitis; Cerebrospinal fluid; Adenosine deaminase.

INTRODUCTION: Tuberculosis remains one of the world's deadliest diseases. Nearly one third of global population, i.e. two billion people, is infected with M. tuberculosis and at risk of developing the disease. Involvement of the central nervous system (CNS) by tuberculosis is the most hazardous type of systemic tuberculosis because of its high mortality rate and possible serious neurological complications and sequelae. It occurs in all age group but $60-70 \%$ of patients are below the age of 20 years. Central nervous system tuberculosis occurs in $2-5 \%$ of all patients with tuberculosis and in $10 \%$ of those with AIDS-related tuberculosis ${ }^{1}$. Co-existence of extra-neural tuberculosis is reported amongst $50 \%$ of cases of neuro -tuberculosis in the literature, which may be a clue to the diagnosis of CNS tuberculosis. CNS tuberculosis, particularly tuberculous meningitis (TBM) remains a serious clinical problem. Missed diagnosis and delayed treatment result in significant mortality and morbidity. The signs and symptoms, results of routine analysis of CSF and radiographic findings for patients with CNS tuberculosis are often inadequate in making a definitive diagnosis. Clearly, prompt laboratory diagnosis is of vital importance. Acid-fast staining of CSF sediment is the most rapid method for detection of mycobacteria, but this method lacks sensitivity. The diagnostic reference standard, isolation of mycobacterium tuberculosis from CSF samples, is insufficiently time (it requires 1-4 weeks) to aid clinical judgement with respect to treatment, and this method is insensitive if large amounts of CSF are not used ${ }^{3}$. The use of polymerase chain reaction (PCR) to 


\section{ORIGINAL ARTICLE}

detect mycobacterium tuberculosis specific DNA may be of potential value. However, the facilities to perform the test are not available in all laboratories and many patients are not affordable because of its high cost. Adenosine deaminase (ADA) is an enzyme of purine catabolism, catalyzing hydrolytic deamination of adenosine to inosine. Detection of high level of ADA has shown promising results in the diagnosis of tuberculous pleural, peritoneal and pericardial effusions ${ }^{4,5}$.

\section{AIMS AND OBJECTIVES:}

1. To study the levels of Adenosine deaminase in cases of meningitis,

2. To evaluate the sensitivity and specificity of CSF-ADA as a diagnostic test in tuberculosis meningitis

MATERIAL \& METHODS: In the cross sectional study, Adenosine deaminase activity (ADA) was studied in cerebrospinal fluid of 60 cases of tuberculous meningitis, 10 cases of pyogenic meningitis. 10 cases of aseptic meningitis and 15 control (patients without any neurological disorders who were given spinal anaesthesia).

Inclusion criteria: 1 . Freshly diagnosed cases of meningitis.

\section{Exclusion criteria:}

1. Old partially treated cases.

2. Some cases whose clinical picture is similar to meningitis like subarachnoid haemorrhage, cerebral malaria were excluded after relevant investigations.

METHODOLOGY: Cases clinically suspected of meningitis, admitted in department of medicine Rama medical college, Kanpur were selected. Valid set of criteria are used to categorize tuberculous meningitis patients including clinical examination, CSF analysis (biochemical, cytological), CT scan (optional), and CSF-ADA activity was estimated in all cases spectrophotometrically and their levels were compared. All the cases were thoroughly examined clinically and investigated.

RESULTS AND DISCUSSION: In our study, we enrolled patients who are diagnosed to have meningitis for the first time. Old and partially treated cases were excluded from the study. CSF-ADA level was estimated and compared .Adenosine deaminase called ADA is an enzyme of purine catabolism which catalyses the pathway from adenosine to inosine. It has got important role in differentiation \& proliferation of lymphoid cells. Specific activity of these enzymes is high in $\mathrm{T}$ lymphocytes than in B cells. The infections like tuberculosis where immunity is cell mediated, enzyme activity is elevated.The results hereby are discussed under separate headings for each variable. 


\section{ORIGINAL ARTICLE}

Table-1: CSF ADA levels in different groups

\begin{tabular}{|c|c|c|c|c|}
\hline \multirow{2}{*}{ Group } & No. of & \multicolumn{3}{|c|}{ CSF ADA Levels (U/L) } \\
\cline { 3 - 5 } & cases & Mean & SD & Range \\
\hline A-TB meningitis & 60 & 14.1 & 1.96 & $10.2-17.2$ \\
\hline B-Pyogenic meningitis & 10 & 4.92 & 1.27 & $3-10.1$ \\
\hline C-Aseptic meningitis & 10 & 3.66 & 1.03 & $1.2-4.9$ \\
\hline D-controls & 15 & 1.69 & 0.44 & $1-2.5$ \\
\hline
\end{tabular}

A Vs B, $\mathrm{t}=13.84, \mathrm{df}=38, \mathrm{p}<0.001$

A Vs $C, t=18.71 \mathrm{df}=42 \mathrm{p}<0.001$

A Vs $D, t=24.02 \mathrm{df}=43 \mathrm{p}<0.001$

Mean CSF ADA levels in tuberculous meningitis groups are significantly higher as compared to pyogenic meningitis, aseptic meningitis and control groups.

Table-2: CSF ADA levels in TB Meningitis in relation to CSF culture

\begin{tabular}{|c|c|c|c|c|}
\hline \multirow{2}{*}{ CSF Culture } & No. of & \multicolumn{3}{|c|}{ CSF ADA Levels (U/L) } \\
\cline { 3 - 5 } & cases & Mean & SD & Range \\
\hline Positive & 8 & 14.4 & 1.96 & $10.2-17.2$ \\
\hline Negative & 52 & 14.45 & 2.20 & $11.8-16.5$ \\
\hline
\end{tabular}

$\mathrm{t}=0.38, \mathrm{df}=28 \mathrm{p}>0.05$

There is no statistical difference in the CSF-ADA levels in culture positive and culture negative tuberculosis meningitis levels in culture positive and culture negative tuberculous meningitis cases.

Table-3: CSF ADA levels in TB meningitis in relation to CSF proteins

\begin{tabular}{|c|c|c|c|c|}
\hline & No. of & \multicolumn{3}{|c|}{ CSF ADA Levels (U/L) } \\
\cline { 3 - 5 } CSF Protein (mg\%) & cases & Mean & SD & Range \\
\hline A: $0-100$ & 16 & 11.76 & 0.99 & $10.2-13.5$ \\
\hline B: $101-200$ & 22 & 14.06 & 1.35 & $12.4-16.5$ \\
\hline C: $>200$ & 22 & 15.82 & 0.99 & $14.8-17.2$ \\
\hline
\end{tabular}

A Vs $B, t=5.22, d f=17, p<0.001$

A Vs C, $\mathrm{t}=11.32 \mathrm{df}=17 \mathrm{p}<0.001$

$B$ Vs $\mathrm{C}, \mathrm{t}=4.14 \mathrm{df}=20 \mathrm{p}<0.001$

CSF-ADA levels were significant in relation to CSF protein. 
Table-4: CSF-ADA levels in TB meningitis in relation to CSF lymphocyte

\begin{tabular}{|c|c|c|c|c|}
\hline & No. of & \multicolumn{3}{|c|}{ CSF ADA Levels (U/L) } \\
\cline { 3 - 5 } Lymphocyte count(\%) & cases & Mean & SD & Range \\
\hline A: $0-50$ & 6 & 10.8 & 0.65 & $10.2-11.5$ \\
\hline B: $51-70$ & 10 & 12.66 & 0.79 & $11.8-13.5$ \\
\hline C: $71-90$ & 24 & 14.72 & 1.32 & $11.8-16.5$ \\
\hline D: $91-100$ & 20 & 15.05 & 1.91 & $12.4-17.2$ \\
\hline
\end{tabular}

A Vs $B, t=7.24, d f=7, p<0.001$

A Vs C, $\mathrm{t}=7.11 \mathrm{df}=13 \mathrm{p}<0.001$

B Vs D, $\mathrm{t}=5.83 \mathrm{df}=11 \mathrm{p}<0.001$

Direct correlation is found between the CSF lymphocyte count and ADA levels.

Table-5: Sensitivity, Specificity and positive predictive value for CSF ADA in diagnosis of tuberculous meningitis.

\begin{tabular}{|c|c|c|c|c|c|}
\hline $\begin{array}{c}\text { Cut off } \\
\text { value }\end{array}$ & $\begin{array}{c}\text { TBM group } \\
(\mathrm{n}=30)\end{array}$ & $\begin{array}{c}\text { Non-TBM } \\
\text { group } \\
(\mathrm{n}=35)\end{array}$ & Sensitivity & Specificity & $\begin{array}{c}\text { Positive } \\
\text { predictive } \\
\text { value }\end{array}$ \\
\hline$>10 \mathrm{U} / \mathrm{L}$ & 60 & 1 & $100 \%$ & $97.44 \%$ & $96.77 \%$ \\
\hline
\end{tabular}

Taking $10 \mathrm{U} / \mathrm{L}$ as the cut-off value of CSF ADA activity and comparing with CSF ADA levels in controls, a sensitivity of $100 \%$ specificity of $97.44 \%$ with a positive predictive value of $100 \%$ is obtained for this test.

CONCLUSION: The mean CSF-ADA value in tuberculous meningitis was statistically significant $(\mathrm{p}<0.001)$ when compared to the CSF-ADA values in pyogenic meningitis, aseptic meningitis and controls. CSF ADA levels correlated with CSF lymphocyte count and protein levels but not with sugar levels. Taking the cutoff value of $10 \mathrm{U} / \mathrm{L}$, the CSF-ADA estimation in tuberculous meningitis has a sensitivity of $100 \%$ and specificity of $97.44 \%$ in the present study.ADA activity in CSF is a rapid, relatively inexpensive and easy procedure, can be of great value in the early diagnosis of tuberculous meningitis

\section{BIBLIOGRAPHY:}

1. Bernaerts A, Vanhoenacker FM, Parizel PM et al. Tuberculosis of the central nervous system: Overview of neuro radiological findings. Eur Radiol 2003; 13: 1876-1890.

2. Radhakrishnan K, Kishore A, Mathuranath PS. Neurological tuberculosis. In: Sharma SK ed. Tuberculosis, 1st Ed., New Delhi; Jaypee Brothers, 2001: PP 209-228.

3. Kennedy DH, Fallon RJ. Tuberculosis meningitis. JAMA 1979; 241:264-8.

4. Marinez Vazquez JM, Ribra e, Ocana I, Sengur RM, Serrat R and Sagrista J. Adenosine deaminase activity in tuberculous pericarditis. Thorax 1986; 41: 888.

5. Sinha PK, Sinha BB and Sinha ARS. Diagnosing tuberculous pleural effusion: Comparative sensitivity of mycobacterial cultural, histopathology and ADA activity. JAPI 1985; 33: 64. 


\section{ORIGINAL ARTICLE}

6. Rich AR, McCordick HA. The pathogenesis of tuberculous meningitis. Bulletin of John Hopkins Hospital, 1933; 52: 5-13.

7. Piras MA and Gakis C. Cerebrospinal fluid adenosine deaminase activity in tuberculous meningitis. Enzyme 1973; 14: 311.

8. McGregar A L. THE MENINGES AND CSF: A synopsis of surgical anatomy, 11th Edn. II; 5-10: 1975.

9. Murray C J L, Feechem RG. Adult mortality in the developing world.

\section{AUTHORS:}

1. Manish Kumar Singh

2. K. Chandran

3. Shrawan Kumar

4. Pranjal Pankaj

5. Ishan Parasher

\section{PARTICULARS OF CONTRIBUTORS:}

1. Assistant Professor, Department of Medicine, Rama Medical College Hospital \& Research Centre, Mandhana, Kanpur, Uttar Pradesh.

2. Professor, Department of Medicine, Rama Medical College Hospital \& Research Centre, Mandhana, Kanpur, Uttar Pradesh.

3. Associate Professor, Department of Medicine, Rama Medical College Hospital \& Research Centre, Mandhana, Kanpur, Uttar Pradesh.
4. Assistant Professor, Department of Medicine, Rama Medical College Hospital \& Research Centre, Mandhana, Kanpur, Uttar Pradesh.

5. Junior Resident, Department of Biochemistry, Rama Medical College Hospital \& Research Centre, Mandhana, Kanpur, Uttar Pradesh.

\section{NAME ADRRESS EMAIL ID OF THE CORRESPONDING AUTHOR:}

Dr. Manish Kumar Singh, House No. 206, Staff Quarter - 2, Rama Medical College, Mandhana, Kanpur, Uttar Pradesh - 209217.

Email - dr.manishkumarsingh@yahoo.com

Date of Submission: 02/08/2013. Date of Peer Review: 03/08/2013. Date of Acceptance: 03/08/2013. Date of Publishing: 07/08/2013. 